\title{
EMPRESARIAS MALAGUEÑAS DE PYMES Y 'SOCIAL MEDIA OPTIMIZATION' (OPTIMIZACIÓN DE LOS MEDIOS DE COMUNICACIÓN SOCIALES)
}

\author{
Ana María Belmonte Jiménez: Universidad de Málaga. España \\ anabelmonte@uma.es
}

\section{RESUMEN}

En la Jornada "Buenas prácticas de empresas laborales constituidas por mujeres socias trabajadoras", que tuvo lugar el 24 de febrero de 2010 en Málaga, las empresarias de pymes comentaban la importancia del uso de las tic en la empresa, mediante el uso de las redes sociales, el marketing y la comunicación. Algunos estudios como los de Cocktail Análisis (2010) configuran un perfil de las mujeres con respecto a la tecnología que va casi siempre por detrás del hombre; en el presente artículo, con respecto al "Social Media Optimization" y las mujeres, y tomando como muestra las entrevistas realizadas a cinco empresarias, se investiga sobre si dicha concepción de la mujer y las tecnologías se corresponde con la realidad. A tenor de los resultados obtenidos por las empresarias entrevistadas, las páginas web de las empresarias tienen una baja implementación del "Social Media Optimization". El desconocimiento del $\mathrm{SMO}$, sus herramientas y sus beneficios son las causas principales citadas por las cuales las empresarias no implementan el SMO.

PALABRAS ClAVE: Social Media Optimization - Empresarias - Pymes - RSS - Medios sociales.

\footnotetext{
1Autor correspondiente:

Ana María Belmonte Jiménez: Becaria de investigación en la Facultad Ciencias de la Comunicación de la Universidad de Málaga, España. 


\title{
MALAGUEÑAS SME CEOS AND SOCIAL MEDIA OPTIMIZATION
}

\begin{abstract}
At the conference "Good practices of companies consisting of women labor working partners", which took place on February 24, 2010 in Malaga, SME CEOs commented on the importance of ICT in the business, using networks social, marketing and communication. Some studies such as Cocktail Analysis (2010) form a profile of women with respect to technology that will almost always behind the man in this article, with respect to Social Media Optimization and women, and taking as example the five interviews with entrepreneurs, we investigate whether this conception of women and technology corresponds with reality. According to the results obtained by the entrepreneurs interviewed, the websites of women CEOs have low implementation of the "Social Media Optimization". Ignorance of the SMO, their tools and their benefits are the main causes cited by women CEOs which do not implement the SMO.
\end{abstract}

KEY WORDS: Social Media Optimization - Entrepreneur women - SME - RSS - Social Media.

\section{INTRODUCCIÓN}

En 2005, Rohit Bhargava inventó el concepto de "social media optimization" en su bitácora, y desde entonces es extensamente conocido en la industria del marketing por la creación de dicho concepto.

Rohit Bhargava (2005) resume el concepto "SMO" (social media optimization) así:

"The concept behind $\underline{S M O}$ is simple: implement changes to optimize a site so that it is more easily linked to, more highly visible in social media searches on custom search engines (such as Technorati), and more frequently included in relevant posts on blogs, podcasts and vlogs."

Rohit Bhargava (2005)

La siguiente investigación está enfocada en el estudio del "social media optimization" $(\mathrm{SMO})$ con un enfoque de género, es por ello que se realizaron cinco entrevistas estructuradas en referencia a los usos de las tácticas y herramientas del "SMO" por parte de cinco empresarias malagueñas.

\section{METODOLOGÍA}

Primero, para conocer el estado de la cuestión sobre el "social media optimization" $(\mathrm{SMO})$ se procedió a la recopilación de diversas fuentes de información, a través de fuentes secundarias (estudios, informes). Después se procedió a entrevistar a cinco empresarias malagueñas sobre el "SMO" en sus páginas webs y sus motivos por los cuáles implementaban sus tácticas y herramientas o los motivos por los cuáles no los implementaban. El tipo de entrevista realizada a las empresarias fue estructurada. Los resultados de dichas entrevistas se reflejan a su vez en una tabla con las direcciones 
web de las empresas. Mediante un análisis de contenido de dichas webs en base a las respuestas afirmativas o negativas de las empresarias sobre el uso de las tácticas y herramientas del SMO se ha realizado dicha tabla.

\section{ANÁLISIS Y DISCUSIÓN}

\subsection{Social Media Optimization (SMO).}

En 2005, Rohit Bhargava inventó el concepto de "social media optimization" en su blog, y desde entonces es extensamente conocido en la industria del marketing por la creación de dicho concepto.

Rohit Bhargava (2005) resume el concepto “SMO” así:

"The concept behind $\underline{S M O}$ is simple: implement changes to optimize a site so that it is more easily linked to, more highly visible in social media searches on custom search engines (such as Technorati), and more frequently included in relevant posts on blogs, podcasts and vlogs."

"El concepto SMO es simple: implementar cambios para optimizar una web para que sea más fácil de enlazar, más visible en los buscadores de medios sociales (tales como Technorati) y sea más frecuentemente incluido en posts relevantes en blogs, podcasts y videoblogs" (traducción propia).

En el año 2009 Rohit Bhargava ha sido nombrado como uno de los asiáticos más influyentes de la comunicación y el marketing. Asimismo, es miembro pionero de la fundación del equipo "360 Digital Influence" de la agencia publicitaria Ogilvy, es escritor de éxito de numerosos libros y artículos, conferenciante y profesor universitario de la asignatura "Global Communications" en la universidad Georgetown de Washington DC. Anteriormente a esto, Rohit dirigió un departamento de publicidad interactiva en Leo Burnett Advertising Agency in Sydney, Australia, donde vivió cinco años. Su blog se encuentra entre los cincuenta mejores blogs de marketing a nivel mundial, y precisamente en su blog es donde lanzó la creación del concepto SMO.

Según la wikipedia (2010), "SMO" "es un conjunto de métodos para atraer visitantes a una web mediante la promoción a través de los medios sociales; es una parte del marketing en medios sociales o "Social Media Marketing", que se dedica a promocionar y publicitar productos y servicios a través de los medios sociales". Se considera uno de los dos únicos métodos de optimizar una web (el otro método sería el Search Engine Optimization -SEO-, que forma parte del search engine marketing). También se considera parte del ORM (online reputation management). Los profesores de la Universidad de Jaén Juan Manuel Maqueira y Sebastián Bruque (2009) en su libro "Marketing 2.0" lo definen como "el concepto que establece las medidas necesarias que un portal corporativo debe tener en cuenta para adaptarse al espíritu de la web 2.0". 
En la versión inglesa de la definición de SMO en la wikipedia (2010) resumen cómo realizar SMO en dos bloques:

1. A través de RSS feeds, social news, botones de compartir, valoraciones del usuario, herramientas de sondeo, e incorporando fotos y video.

2. Realizar actividades promocionales en medios sociales publicando contenidos, tal y como puede ser mediante la creación de un blog, insertando comentarios en otros blogs, participando en discusiones de grupos y creando y actualizando la información en perfiles de redes sociales (publicidad no convencional) (traducción propia).

Rohit Bhargava (2005), el creador del término, lanzó las primeras cinco reglas del "SMO" que posteriormente fueron ampliadas por otros colaboradores, conformando un total de dieciséis reglas.

Oscar Ugaz(2006), digital business manager del Real Madrid Club de futbol y consultor en marketing y nuevas tecnologías, en su blog sobre dichos temas, hace una traducción sobre los principios del SMO que aparece como traducción española del concepto SMO en el propio blog de Rohit Bhargava.

Según esta traducción española de Óscar Ugaz, estas serían las primeras cinco reglas del SMO propuestas por Rohit:

Regla 1. Incremente su capacidad de ser enlazado: La primera y más importante. La página web en lo posible tiene que dejar de ser "estática" - es decir que raramente es actualizada y solo es usada como "folleto informativo online". Agregar un blog es un gran paso. Sin embargo, hay otras formas como crear casos de estudio o similares o aun más simple: agregar contenido que ya existe en otro lugar en un formato fácil de usar (RSS por ejemplo). Hay que matizar que cuando el creador del concepto "Social Media Optimization" Rohit Bargava escribió esto escribió esto (en el año 2005) apenas habían sido recién creadas las redes sociales como facebook, por lo que al principio la función de estas redes era meramente social y pocas empresas creaban sus páginas o grupos, ya que aún no se vislumbraba ésta utilidad. Si actualizamos la situación, al ejemplo del blog tendríamos que añadirle el de los perfiles sociales en medios como redes sociales, microblogs, canales de video, canales de fotos... así como los botones de acceso que se pueden incluir en la web para acceder a dichos perfiles. También podemos agregar contenido que ya existe en otro lugar en un formato fácil de usar ("RSS”) para que los públicos accedan de manera periódica a las actualizaciones del portal.

Regla 2. Facilite que su página se agregue a favoritos o acepte tags: Agregar botones para marcadores sociales como "del.icio.us" u otro servicio de "bookmarking" similar es una de las formas más sencillas de facilitar el proceso de "tagging" (etiquetado).

Regla 3. Premie a los que incluyan su web como un link en sus propias páginas / blogs (inbound links): Usados como el barómetro para medir el éxito de un blog (y 
por qué no, también el de una página web), los "inbound links" (links recíprocos) son la vía para mejorar la posición en resultados de búsquedas o cualquier otro ranking. Para incitar su uso, necesitamos hacer el proceso sencillo y dar brindar beneficios claros. El uso de "Enlaces permanentes" (es decir, una dirección especifica URL para cada una de las páginas de nuestra web o en el caso de los blogs, la dirección de cada post) y la facilidad con que estas direcciones especificas pueden ser "copiados" y "pegadas", resuelve el primero de los puntos. Los beneficios para aquellos que nos incluyan como links pasa por acciones tan simples como listarlos en nuestro "site" o web y así brindarles visibilidad también a ellos. "Quid pro quo".

Regla 4. Ayude a que su contenido "viaje": A diferencia del "SEO" (search engine optimization, optimización de webs en buscadores), "SMO" no trata únicamente de hacer ajustes a una página web. Si tiene contenido que pueda ser "portable" (como por ejemplo PDFs, files de video o audio),envíelos a sites o blogs relevantes. Esto ayudará a que dicho contenido viaje más lejos y finalmente pueda crear atención y links a su página.

Regla 5. Promueva el uso de "mashups" (híbridos): Un concepto muy en boga en Internet 2.0. Se trata básicamente de usar contenido o aplicaciones tomadas de diversos lugares para crear nuevo contenido.

Jeremiah Owyang agregó las reglas 6 y 7 :

Regla 6. Sea una fuente útil para sus usuarios, incluso si no lo ayuda directamente: Agregue valor a sus usuarios incluyendo links a páginas $\mathrm{u}$ información que pueda ayudarlos con sus objetivos y propósitos. Presentado adecuadamente, incluso podrá hacer links a su competencia. De esta formar su página se convierte en el punto de referencia de una comunidad específica. ¿Esto refuerza el concepto de "SMO"? Si la gente "linkea" su página y la marca como útil, mejorará su visibilidad social. A más "tags" o "marcas", su página se vuelve más relevante para los motores de búsqueda.

Regla 7. Premie a los usuarios valiosos y útiles: Este tipo de usuarios serán buenos influenciadores o voceros de su página. Busque formas de "premiarlos". Quizás pueda presentarlos en su página principal, o desarrollar un sistema de "rankeo". Algo que genere atención sobre esas personas. Otras veces un rápido correo electrónico o una nota privada agradeciéndoles su ayuda puede ser muy favorable. Trate de ser siempre sincero. Quizás esto no sea "SMO" per se, pero ayudara a mejorar la fidelidad de su comunidad para con su página web.

Cameron Olthuis añadió las reglas 8,9, 10 y 11:

Regla 8. Participe: Únase a la conversación. Internet se está volviendo una calle de dos sentidos. Al "conversar" con su comunidad está logrando exposición y logrando que la gente hable de usted (procure que siempre lo haga positivamente). Participar hace que su mensaje se extienda más lejos y más rápido. 
Regla 9. Conozca cómo llegar a su audiencia: Si no conoce a su audiencia, está en problemas. Quisiéramos que todo el mundo use nuestro producto, pero hay que ser realista. Siempre habrá una audiencia especifica que lo encontrarán interesante y otros que no. Preocupe de agradar al que de verdad está interesado.

Regla 10. Cree contenido: Hay ciertos contenidos que se extienden socialmente de manera natural, No importa en qué industria se encuentre o que tan aburridos sean los productos que vende, siempre habrá un tipo de contenido que funciona. Quizás sea hacer que la gente se ría, escribir artículos o casos, u ofrecer pequeñas aplicaciones de entretenimiento (screensaver, postales) o algún "widget" (pieza de código que los usuarios pueden agregar a su página web, con forma gráfica y que funciona como una miniaplicación o programa). Averigüe qué tipo de contenido funciona en su caso y créelo.

Regla 11. Sea auténtico: Una comunidad no premia a los que son falsos. Este post puede ser muy ilustrativo al respecto.

Loren Baker incorporó las reglas 12 y 13

Regla 12. No olvide sus raíces, sea humilde: Si se ha convertido en un "BlogStar" o su página obtiene un tráfico impresionante, no permita que se le suban los humos. Recuerde a aquellos que lo ayudaron a estar allí. Mantener ese respeto será positivo para todos los involucrados.

Regla 13. No tenga miedo de probar nuevas cosas, mantenga fresca su propuesta: Internet como fenómeno social muta cada minuto. Esté al tanto de las nuevas herramientas, productos y retos.

Lee Odden agregó las reglas 14, 15 y 16:

Regla 14. Desarrolle una estrategia para el "SMO": defina sus objetivos y establezca metas. Sea totalmente consciente de lo que quiere lograr como resultado de estas tácticas. Puede ser reputación, ventas, influencia, credibilidad, tráfico, páginas vistas, etc.

Regla 15. Escoja sus tácticas inteligentemente: sea consciente de que acciones tendrán más impacto en la búsqueda de sus objetivos.

Regla 16. Haga del "SMO" parte su proceso de mejores prácticas: busque maneras de incorporar las tácticas de "SMO" a su proceso de trabajo, documéntelo y distribúyalo entre los involucrados. Empiece con lo más sencillo (incluya links a otros sites y "bookmarks") y evolucione a partir de allí. 


\subsection{Amesal y las mujeres empresarias.}

Algunos estudios como los de Cocktail Análisis del año 2010 configuran un perfil de las mujeres con respecto a la tecnología (incidiendo en el uso de los medios sociales) que va casi siempre por detrás del hombre. En el presente artículo, con respecto al "SMO" y las mujeres, y tomando como muestra a cinco empresarias pertenecientes a Amesal, se investiga sobre si dicha concepción de la mujer y las tecnologías de corresponde con la realidad.

Amesal es la "Asociación de Mujeres Emprendedoras y Empresarias de Sociedades Laborales". Su principal objetivo es desarrollar acciones que sirvan para favorecer el emprendimiento de las mujeres y para garantizar el desarrollo de su actividad empresarial, eliminando aquellos obstáculos que se les presentan de forma cotidiana y que tienen que ver con sus iniciativas emprendedoras y empresariales. Amesal se constituyó a finales de 2003 con el claro objetivo de ser un referente en Igualdad de Oportunidades de las Sociedades Laborales de Madrid y de servir de agente estratégico en el desarrollo de la acción positiva en la Economía Social.

Ante la llegada de nuevas tecnologías en la comunicación, siempre se suceden posturas apocalípticas o integradas (Eco, 1965).

En la Jornada "Buenas prácticas de empresas laborales constituidas por mujeres socias trabajadoras", a la cual tuve la oportunidad de asistir, las empresarias de pymes comentaban la importancia del uso de las tic en la empresa, de las redes sociales y del marketing y la comunicación.

Las posturas apocalípticas e integradas se comentaban como en un foro de discusión. Amablemente, cinco empresarias accedieron entrevistarse conmigo sobre la implementación del “Social media optimization” en sus páginas web.

Las empresarias entrevistadas fueron las siguientes:

- Manuela Aránega, directora de la agencia de marketing "acción mk" (Málaga)

- Marta Pinazo Sanz, directora de la editorial "Ediciones Aljamar" (Málaga)

- María del Carmen Piedad Herrera, directora de empresas de varios servicios "Papillón" (Marbella, Málaga)

- Marta González, directora de la empresa de estampación “stampate” (Málaga)

- Macarena Gómez, directora de la empresa de gestión medioambiental "Tandemqc". (Málaga)

Previamente, para la realización del cuestionario, las incógnitas que se presentaban eran las siguientes:

¿Conocen las empresarias las técnicas y herramientas del "social media optimization"? ¿Por qué implementan dichas técnicas, si lo hacen, y por qué no, si no lo hacen? 
Las preguntas de las entrevistas eran las siguientes:

- ¿Dispone su página web de "marcadores sociales"?

- ¿Por qué?

- ¿Dispone su página web de "enlaces permanentes"?

- ¿Por qué?

- ¿Dispone su web de "perfiles en medios sociales" ?

- ¿Cuáles?

- ¿Por qué?

- ¿Dispone su web de "contenido difundible" (videos, archivos en pdf, etc.)?

- ¿Dispone su página web de "mashups y widgets"?

- ¿Por qué?

- ¿Dispone su página web de RSS? ¿Por qué?

Los resultados generales sobre la presencia o ausencia de los ítems de la entrevista se exponen en la siguiente tabla:

\begin{tabular}{|c|c|c|c|c|c|c|c|}
\hline WEB DE LA EMPRESA & $\begin{array}{l}\text { Marcadores } \\
\text { sociales }\end{array}$ & $\begin{array}{l}\text { Página } \\
\text { web con } \\
\text { enlaces } \\
\text { perman } \\
\text { entes }\end{array}$ & $\begin{array}{l}\text { medios } \\
\text { sociales }\end{array}$ & $\begin{array}{l}\text { BOTON } \\
\text { ES DE } \\
\text { MEDIO } \\
\text { S } \\
\text { SOCIA } \\
\text { LES }\end{array}$ & $\begin{array}{l}\text { Conteni } \\
\text { do } \\
\text { difundi- } \\
\text { ble }\end{array}$ & $\begin{array}{l}\text { MA } \\
\text { SHU } \\
\text { PS Y } \\
\text { WID } \\
\text { GET } \\
\text { S }\end{array}$ & RSS \\
\hline www.accionmk.com & $\mathrm{NO}$ & SÍ & SÍ & $\mathrm{NO}$ & SÍ & $\mathrm{NO}$ & SÍ \\
\hline www.papillonbylis.com & NO & NO & SÍ & NO & NO & NO & NO \\
\hline www.stampatemalaga.com & NO & $\mathrm{NO}$ & NO & SÍ & SÍ & $\mathrm{NO}$ & NO \\
\hline www.edicionesaljamar.es & $\mathrm{NO}$ & NO & $\mathrm{NO}$ & NO & $\mathrm{NO}$ & NO & NO \\
\hline www.tandemqc.com & NO & $\mathrm{NO}$ & $\mathrm{NO}$ & NO & $\mathrm{NO}$ & $\mathrm{NO}$ & NO \\
\hline
\end{tabular}

Fuente: Elaboración propia. 
Un $45 \%$ de las encuestadas afirman que actualizan su portal cada dos o más meses, otro $45 \%$ de manera anual y sólo un $10 \%$ afirma actualizarla a diario, por lo que vemos que las empresarias entrevistadas no le prestan demasiada atención a la actualización de los contenidos de su portal corporativo. Ninguna de las entrevistadas usan "marcadores sociales" en sus páginas webs. Sólo el 10\% de las encuestadas usa "rss"; el $90 \%$ no lo implanta en su página web. Sólo un 10 \% incluye enlaces permanentes en sus páginas web; el 90 \% restante no los incluye.

Un $40 \%$ de las encuestadas afirman tener su perfil de empresa en facebook. Un $20 \%$ de las encuestadas tiene su canal de video en youtube y otro $20 \%$ tiene su perfil de empresa en Twitter. Sólo un 10\% tiene su perfil en "picassa" (portal para el almacenamiento de fotos) y disponen de un blog.

El motivo de la no presencia en medios sociales que argumentan un $90 \%$ de las encuestadas es el desconocimiento.

Gráfico no 1: Actualización del portal

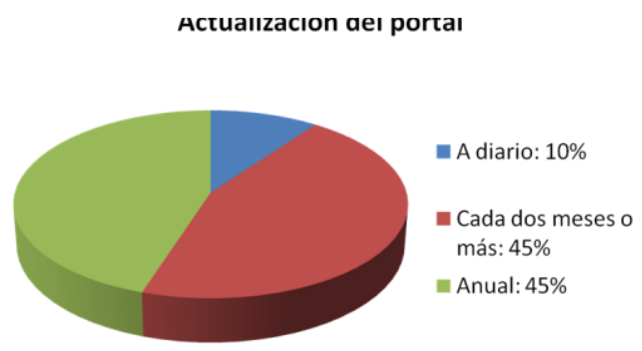

Fuente: Elaboración propia
Gráfico $n^{\circ}$ 2: Perfiles en medios sociales Perfiles en medios sociales

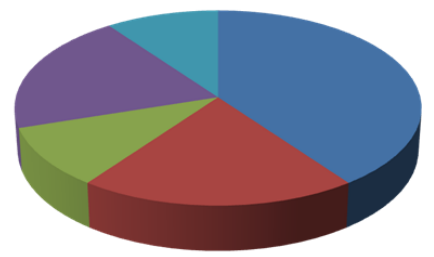

- Facebook: $40 \%$

- You tube: $20 \%$

Inlog: $10 \%$

- Twitter:20\%

- Picassa: $10 \%$

nedes verticales: $0 \%$

Fuente :Elaboración propia

Sólo un $10 \%$ de las encuestadas incorpora "botones" o "íconos" de acceso a los "medios sociales" en su página web; el 90 por ciento restante, pese a tener perfiles en medios sociales, no incorpora en su web los botones de acceso a los mismos.

El 10\% argumenta que no tiene un perfil de empresa en los medios sociales porque piensa que ahí no está su público objetivo. Sólo un 10\% afirma tener su perfil en redes verticales. Ninguna de las encuestadas incorpora a su portal mashups y widgets.

El $80 \%$ de las encuestadas afirman tener en su portal corporativo material que puede ser difundido como informes en pdf, videos... Un $20 \%$ no incorpora a su portal. 
Gráfico no ${ }^{\circ}$ : RSS

RSS

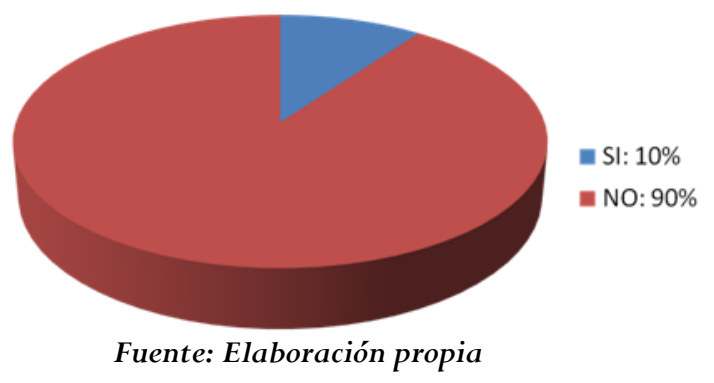

Gráfico n ${ }^{\circ}$ 5: Contenido difundible

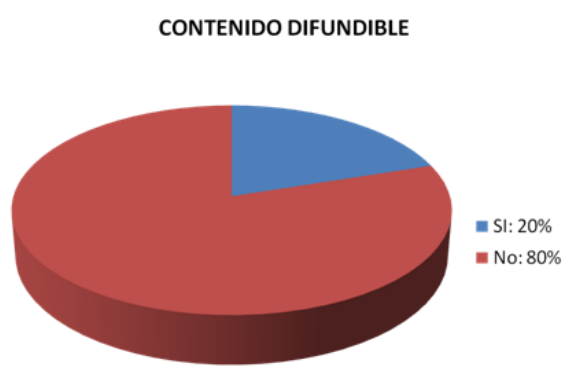

Fuente: Elaboración propia

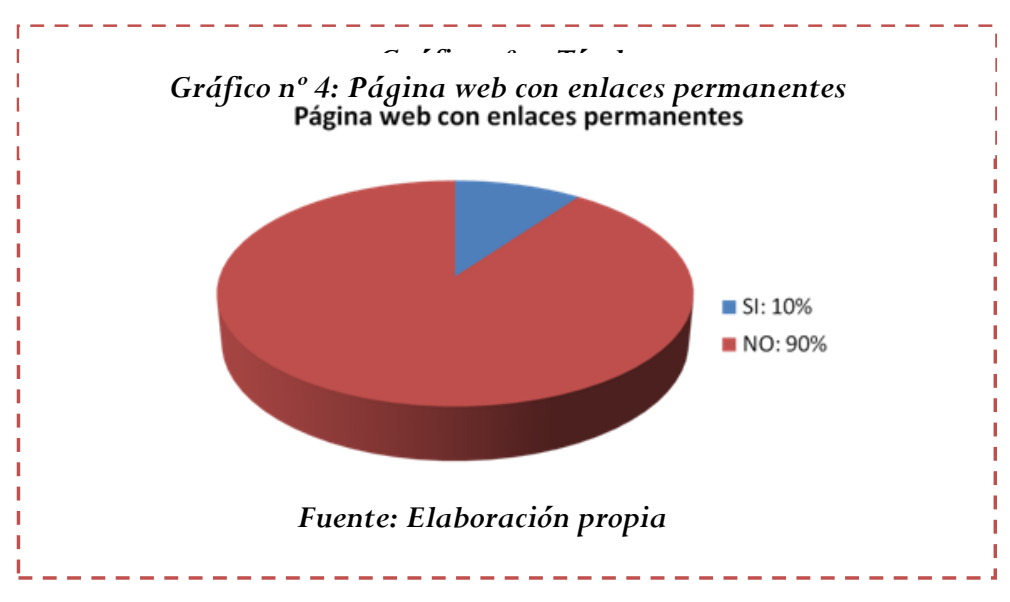

Gráfico ${ }^{\circ}$ 6: Motivos de no presencia en medios sociales Motivos de no presencia en medios sociales

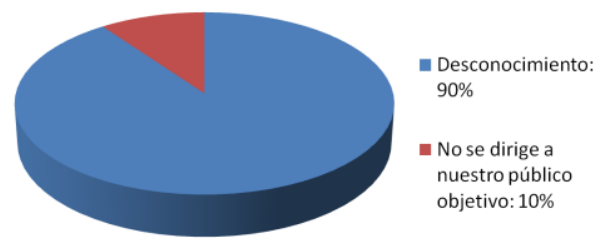

Fuente: Elaboración propia

\section{CONCLUSIONES}

A tenor de los resultados obtenidos por las empresarias entrevistadas, las páginas web de las empresarias tienen una baja implementación del "Social Media Optimization". El desconocimiento del SMO, sus herramientas y sus beneficios son las causas principales citadas por las cuales las empresarias no implementan el SMO.

Las empresarias entrevistadas no aprovechan las oportunidades que les ofrece el "Social Media Optimization, cuyas herramientas y cuya publicidad no les supone a la empresa coste alguno, razón de más para que el presupuesto no suponga un obstáculo en la adopción de dicho método de optimización para los portales corporativos de sus pymes.

Sin embargo, también hay que resaltar, que la gran mayoría de las empresas (ya sean grandes o pequeñas) se encuentran en una primera fase de adopción, que se espera que vaya a más a medida que las empresas sepan aprovechar las herramientas que la denominada web 2.0 y el SMO les ofrece. 
En definitiva tener un portal web optimizado y una buena presencia en los medios sociales, son "la carta de presentación" perfecta para toda empresa que quiera tener una buena presencia en el mundo on line, y el social media optimization, le brinda a toda empresa (al margen de su presupuesto) de dicha oportunidad.

\section{REFERENCIAS}

\section{Bibliografía.}

Eco, U. (1965). Apocalípticos e integrados (Vol. 26204). Editorial de bolsillo.

Maqueira, J. M. y Bruque, S. (2009): Marketing 2.0. El nuevo marketing en la web de las redes sociales. Madrid: RA-MA.

\section{Webgrafía.}

Bhargava, R. “About Rohit Bhargava”. Disponible en:

http://rohitbhargava.typepad.com/socialmediabio/

Rugaz, O. (2006). “SMO: Reglas para la "visibilidad" social de su web". Disponible en: www.oscarugaz.com/2006/09/reglas_para_la_.html

The cocktail analysis (2010): “Observatorio sobre viajes e internet”. Disponible en: http://tcanalysis.com/blog/posts/primera-oleada-del-observatorio-sobre-viajes-einternet

\section{Ana María Belmonte Jiménez}

Becaria del Dpto. de Comunicación Audiovisual y Publicidad en la Facultad Ciencias de la Comunicación de la Universidad de Málaga. Especializada en Nuevas Tecnologías de la Comunicación y en Comunicación de Género. 